\title{
Laminar and Turbulent Boundary Layers on a Shark Fin
}

\author{
Husein Noble, Shrey Kulkarni, Kartik Sunil, Prasad Pokkunuri \\ Mahindra École Centrale \\ Survey No: 62/1A, Bahadurpally, Jeedimetla, Hyderabad, India \\ Husein170318@mechyd.ac.in; shrey170348@mechyd.ac.in; kartiksunil170324@mechyd.ac.in; \\ prasad.pokkunuri@mahindrauniversity.edu.in
}

\section{Extended Abstract}

The research undertaken, focuses on understanding and analysing the drag reducing properties of the shark skin. The surface of the skin of the shark consists of tiny riblets, which are the primary cause of this property. Flow across the sharkskin is turbulent, due to the riblet structure perturbing the laminar flow by providing an obstruction to the streamlines. In a laminar flow, fluid drag increases with an increase in surface area. This is due to the shear stress or viscosity of the fluid acting across the new larger surface area. In the turbulent flow regime of the shark skin, vortices tend to form on the tips of the riblets. However, these remain on the surface of the riblets, and don't cause any high velocity flow in the valleys of the riblets. This low velocity flow and low surface area in the valley of the riblets leads to very low shear stresses, compared to the top of the riblets which do experience a high shear stress due to the faster fluid flow.

The riblets in reality have a complex geometry and thus are difficult to model and so to decrease the complexity various simpler geometrical configurations are used instead like the $\mathrm{V}, \mathrm{L}$ or $\mathrm{U}$ continuous configurations. As flow properties change, the dimensions of the turbulent-flow structures change as well. A non-dimensional length is used to compare results obtained for different flow conditions. The main purpose of this numerical experiment is to determine which of the studied configurations causes minimum drag. Multiple 3D models of riblets are created (as shown in Fig-1) and the resulting flow is computed using appropriate boundary and input conditions and velocity contours are generated. Essentially, an effective contact area leads to increased or reduced drag. With the presence of riblets, the velocity gradient at the bottom of these valleys within the riblets almost vanishes, greatly reducing the wall average velocity gradient. This phenomenon is most prevalent in the triangular V riblets. Once the valleys fill up with almost still water, the flow slips more easily over the ribbed surface. This leads to the immense drag reduction properties of sharks, and help them swim at relatively high speeds in spite of the huge contact area with the water.

This proves our earlier hypothesis that the riblets help slow down velocity of flow, as well as reduce cross-streams and increase the laminar characteristic of the flow. A change in shear percentage corresponds to a more or a less turbulent flow. If there is lower change in the shear stress, the flow is less turbulent and if there is a higher change in the shear, there is a stronger turbulent characteristic. Here we can see the riblets play a role in reducing drag in the flow, by reducing the flow velocity of the fluid and hence reducing the drag of the same. It is observed that the change in shear percentage is the least for a particular non-dimensional constant (Non-dimensional riblet spacing). It is seen that triangular riblets are most efficient in slowing down the fluid. The contour for the triangular riblets have a slower velocity flow than the other shapes, as well as the lowest shear stress gradient. Hence, these seem to be the best shape of riblets in helping reduce the drag coefficient of the overall flow.

\section{References}

[1] Dean et al, "Shark-skin surfaces for fluid-drag reduction in turbulent flow", 2010, Philosophical Transactions of Royal Society, 368, 4775-4806.

[2] Bai et al, "Marine drag reduction of shark skin riblet surfaces", 2017. Science Direct Biotribology 11-24.

[3] Lang et al. "Shark skin boundary layer control", 2012. Natural Locomotion in Fluids, IMA 155, DOI 10.1007/978-14614-3997-4_9

[4] Frank M.White, Fluid Mechanics, McGraw Hill Publications, 2017 